\title{
Segmentation-Based Regularization of Dynamic SPECT Reconstruction
}

\author{
T. Humphries, A. Saad, A. Celler, G. Hamarneh, T. Möller, and M.R. Trummer.
}

\begin{abstract}
Dynamic SPECT reconstruction using a single slow camera rotation is a highly underdetermined problem, which requires the use of regularization techniques to obtain useful results. The dSPECT algorithm (Farncombe et al. 1999) provides temporal but not spatial regularization, resulting in poor contrast and low activity levels in organs of interest, due mostly to blurring. In this paper we incorporate a user-assisted segmentation algorithm (Saad et al. 2008) into the reconstruction process to improve the results. Following an initial reconstruction using the existing dSPECT technique, a user places seeds in the image to indicate regions of interest (ROIs). A random-walk based automatic segmentation algorithm then assigns every voxel in the image to one of the ROIs, based on its proximity to the seeds as well as the similarity between time activity curves (TACs). The user is then able to visualize the segmentation and improve it if necessary. Average TACs are extracted from each ROI and assigned to every voxel in the ROI, giving an image with a spatially uniform TAC in each ROI. This image is then used as initial input to a second run of dSPECT, in order to adjust the dynamic image to better fit the projection data.

We test this approach with a digital phantom simulating the kinetics of Tc99m-DTPA in the renal system, including healthy and unhealthy behaviour. Summed TACs for each kidney and the bladder were calculated for the spatially regularized and nonregularized reconstructions, and compared to the true values. The TACs for the two kidneys were noticeably improved in every case, while TACs for the smaller bladder region were unchanged. Furthermore, in two cases where the segmentation was intentionally done incorrectly, the spatially regularized reconstructions were still as good as the non-regularized ones. In general, the segmentation-based regularization improves TAC quality within ROIs, as well as image contrast.
\end{abstract}

Index Terms-dynamic SPECT, dSPECT, image reconstruction, segmentation, random walk

\section{INTRODUCTION}

The goal of dynamic single photon emission computed tomography (SPECT) is to reconstruct a time series of threedimensional images from a SPECT scan. From these images one can extract information about the temporal behaviour of the radiotracer in order to assess bodily function; for instance, the metabolism of ${ }^{99 \mathrm{~m}} \mathrm{Tc}-\mathrm{DTPA}$ by the renal system. A dynamic SPECT reconstruction can provide better quantitative information than current planar dynamic nuclear medicine techniques, which are not able to accurately correct

Thomas Humphries (thomashesfu.ca) and Manfred Trummer are with the Department of Mathematics, Simon Fraser University, Burnaby, British Columbia, Canada, V5A 1 S6.

Ahmed Saad, Ghassan Hamarneh and Torsten Möller are with the Department of Computer Science, Simon Fraser University, Burnaby, British Columbia, Canada, V5A 1 S6.

Anna Celler is with the Department of Radiology, University of British Columbia, Vancouver, British Columbia, Canada, V5Z 1L8. for effects due to attenuation and organ overlap, due to their two-dimensional nature.

A reconstruction method using a conventional single slow camera rotation is desirable, as it is easily implemented with current technology. A drawback of this approach, in which each projection corresponds to one timeframe of the 4D reconstructed image, is that it results in a highly underdetermined reconstruction problem. In such a problem there are many more unknown values to be solved for than there are equations that constrain them, meaning that ther are infinitely many possible solutions. As a result, one must apply regularization techniques to obtain a physiologically realistic solution. The dSPECT method of Farncombe et al. [1], [2] imposes temporal regularization by forcing the time-activity curve (TAC) in each voxel to obey a simple constraint. In this paper, we incorporate a user-assisted image segmentation method [3] into the dSPECT reconstruction process to provide additional spatial regularization. This segmentation-based regularization improves TAC accuracy and image contrast.

\section{Methodology}

A digital phantom was used to model tracer kinetics in the renal system, including kidneys, ureters, bladder and background activity. Time-dependent activity concentrations were generated using a compartmental model (Fig. 1, Table I), and then assigned to anatomical regions obtained from the NCAT [4] digital phantom. A $370 \mathrm{MBq}$ injection was simulated, with $20 \%$ going to the renal system and the rest distributed evenly as background. Six different test cases were simulated: (A) healthy behaviour in both kidneys, (B) unhealthy behaviour in both kidneys, (C) unhealthy behaviour in the left kidney, (D) unhealthy behaviour in the right kidney, (E) unhealthy behaviour in only the bottom third of the left kidney, and (F) unhealthy behaviour in the bottom third of the right kidney.

Healthy renal behaviour was defined as wash-in and washout with peak activity occurring 100s post-injection and $T_{\frac{1}{2}}$ (half-maximum of activity, during washout) at 290s. Unhealthy behaviour had peak activity at 200s and $T_{\frac{1}{2}}$ at 2135s. A set of projections was generated from the phantom, including the effects of Poisson noise, attenuation and collimator blurring. The acquisition protocol was a dual-head rotation over $270^{\circ}$ per head, with the two heads at $90^{\circ}$ to one another. The simulated rotation started with one head behind the supine patient and the other next to the right kidney, with the heads rotating clockwise. This protocol was chosen because it provides spatial information for each time frame from two 


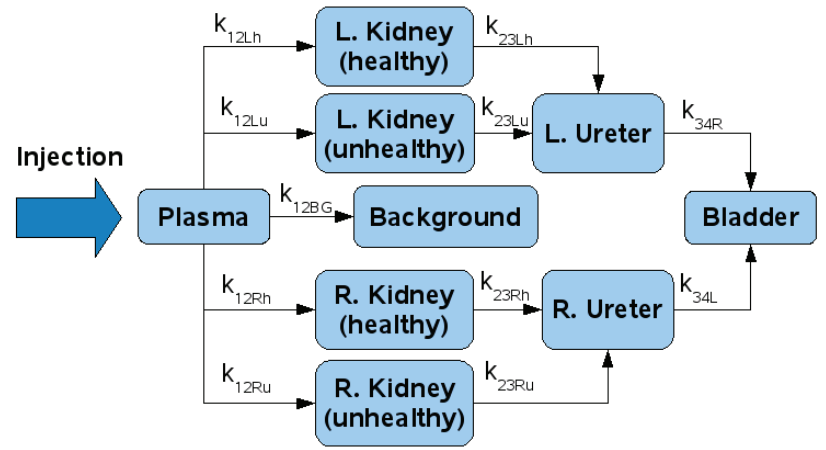

Fig. 1. Generalized compartmental model used to generate TACs for the different anatomical regions in the dynamic digital phantom. Transfer coefficients $k$ were altered to model healthy or unhealthy behaviour (See Table I). Depending on the situation, some compartments were not used; for instance, phantoms A, C and E did not include the compartment for unhealthy right kidney.

\begin{tabular}{|l|c|c|c|c|}
\hline & $k_{12 R}, k_{12 L}$ & $k_{12 B G}$ & $k_{23 R}, k_{23 L}$ & $k_{34 R}, k_{34 L}$ \\
\hline Healthy (h) & 0.2 & 1.6 & 0.3 & 1.0 \\
Unhealthy (u) & 0.2 & 1.6 & 0.03 & 0.1 \\
\hline
\end{tabular}

TABLE I

KINETIC COEFFICIENT VALUES USED IN THE COMPARTMENTAL MODEL (FIG. 1) TO GENERATE THE HEALTHY AND UNHEALTHY TIME-DEPENDENT ACTIVITY IN EACH ORGAN. THESE VALUES WERE CHOSEN BECAUSE THEY PROVIDED CURVES THAT AGREED WELL WITH PLANAR CLINICAL DATA. UNHEALTHY BEHAVIOUR WAS SIMULATED BY KIDNEY-TO-URETER AND URETER-TO-BLADDER COEFFICIENTS THAT WERE 10\% THE VALUE OF THE HEALTHY COEFFICIENTS.

different angles, and because it acquires less attenuated data from behind the patient early in the scan, when tracer kinetics are changing more rapidly. Forty-eight 20 s projections were simulated.

The projections and attenuation map generated from the phantom were used as input to the dSPECT [1] reconstruction algorithm. dSPECT is an iterative expectation-maximization (EM) algorithm which enforces a simple constraint on the TAC for each image voxel - the activity must only increase, only decrease, or increase to a peak and then decrease over the whole time interval. This constraint is enforced through the inclusion of a difference tensor, $A$, into the standard maximum-likelihood expectation maximization (MLEM) [5] algorithm. Rather than optimizing over image intensities $x$, the algorithm optimizes over the temporal differences between neighbouring time frames, denoted by $\tilde{x}=A x$. Since all the differences in $\tilde{x}$ are positive, the desired temporal behaviour is enforced. Thus, the acquisition is modelled by

$$
C A^{-1} \tilde{x}=p
$$

where $C$ is the projection system matrix including patientspecific attenuation and depth-dependent collimator resolution modelling, $A^{-1}$ is the inverse of $A$, and $p$ is the acquired projection data, including Poisson noise. $A^{-1}$ is guaranteed to exist since the mapping from $x$ to $\tilde{x}$ is one-to-one. The
dSPECT update formula is then given by

$$
\tilde{x}_{i k}^{\text {new }}=\frac{1}{\sum_{j} C_{i j k} A_{i j k}^{-1}} \tilde{x}_{i k}^{\text {old }} \sum_{j} C_{i j k} A_{i j k}^{-1} \frac{p_{j k}}{\sum_{i} C_{i j k} A_{i j k}^{-1} \tilde{x}_{i k}^{\text {old }}}
$$

where $i$ and $j$ are matrix/vector (spatial) indices, and $k$ is the temporal index (time frame).

The number of time frames $k$ corresponds to the number of projection views acquired - forty-eight in this study. Since the time frame in which the maximum TAC value occurs is not known a priori, the algorithm also allows the assumed peak location for each voxel (encoded in $A$ ) to shift after every iteration, based on the projection data [2]. For instance, if it is initially assumed that activity in a voxel peaks in the $16^{\text {th }}$ time frame, but the projection data suggest that it should peak earlier, then the assumed peak location will shift to an earlier time frame. Due to the necessity of locating the peak location for each voxel, the algorithm must be run with more iterations than are conventionally used for static MLEM to obtain a satisfactory reconstruction.

dSPECT was run with sixty iterations to generate a spatially non-regularized (NR) reconstruction for each test case. These reconstructions were computed beginning with a generic initial condition where activity was assumed to be increasing in the lower 24 slices of the volume (containing only background and bladder) and assumed to peak at the $24^{\text {th }}$ time frame in the upper 40 frames (which contained the kidneys). This initial condition and number of iterations were chosen based on earlier experiments where they gave good results. The (NR) reconstructions gave a reasonable approximation to the true activity, but suffered from poor contrast between the organs of interest and background activity, as well as a lack of uniformity within each region of interest (ROI). The poor contrast resulted in lowered activity levels in the organs of interest, as their boundaries blurred with the background. To address this problem, we incorporated segmentation into the reconstruction algorithm.

Segmentation was achieved by a user-assisted method [3], as follows. Each (NR) reconstruction was loaded into an interactive segmentation program and a single coronal slice that showed all ROIs (the two kidneys and the bladder) was examined. A user placed seeds for five segments (zero activity, background activity, right kidney, left kidney and bladder) and then ran an automatic 4D probabalistic segmentation algorithm. Fig. 2 shows an example of the seeding procedure. In cases $\mathrm{E}$ and $\mathrm{F}$ a sixth segment was also seeded in the unhealthy part of one kidney. The algorithm generated a probabilistic field using a random walk approach [6] that took into account both the proximity of each voxel to the user-defined seeds, as well as similarity between TACs in each voxel within a segment. Each voxel was thus assigned a probability of belonging to each of the segments, and made part of the segment corresponding to the highest probability.

Once the automatic segmentation was completed, the user was able to visualize the probabilistic segmentation result using volume rendering [7]. Then, the user could interact in real-time with the probabilistic field through intuitive graphical user interface widgets to improve the final segmentation. For example, if voxels that were clearly part of the left 

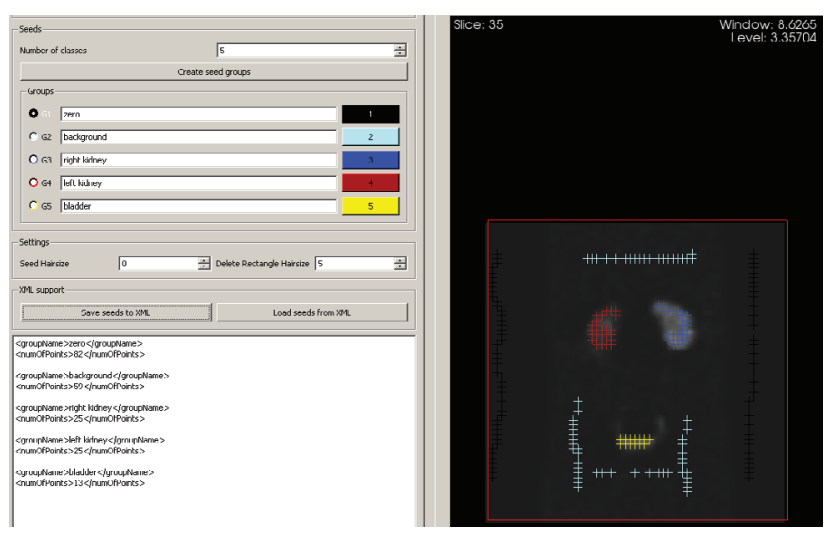

Fig. 2. Screenshot of the interactive segmentation program that was used in the proposed reconstruction method, illustrating typical user seeds (indicated by coloured ' + ' signs in the right panel) that were placed before running the probabilistic segmentation. The five segments are zero activity (black), background (cyan), right kidney (blue), left kidney (red) and bladder (yellow)

kidney were assigned to the right kidney segment due to the similarity between TACs, they could be re-assigned to the left kidney segment. Fig. 3 gives an example of a volume-rendered segmentation obtained after this step. The segmentation was obtained from the (NR) reconstruction only, with no additional knowledge about organ locations (e.g. from a CT map), aside from the seeds provided by the user.

Average TACs for each segment were computed, and then assigned to every voxel in that segment, creating an averaged image where the TAC was spatially uniform in every segment. This image was used as the initial input for another run of dSPECT, this time over 50 iterations. The purpose of the second run was to adjust the segmented image to better fit the projection data. This is necessary since in a realistic situation one would not expect the activity within each segment to be perfectly uniform; furthermore, this step corrects for errors made during the segmentation process, such as assigning regions with different behaviour to the same segment and then averaging them. The final result of this calculation was our

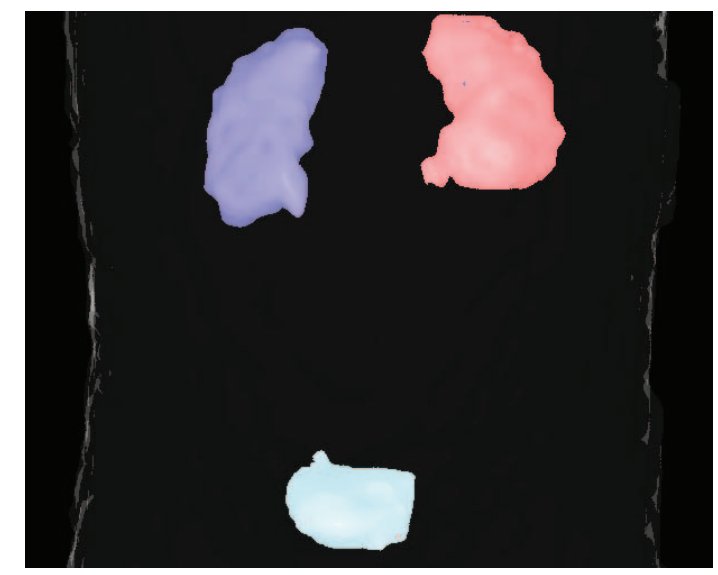

Fig. 3. 3D volume rendering of segmentation obtained for case C, showing right kidney (blue), left kidney (red), bladder (cyan) and background (grey boundary).

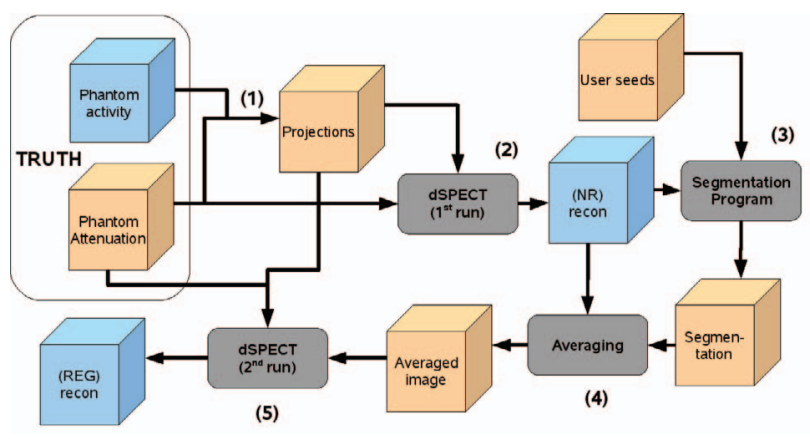

Fig. 4. Flowchart of calculations performed in the study. (1) Creation of projections from phantom volumes, (2) Creation of (NR) reconstruction using dSPECT, (3) Segmentation of (NR) reconstruction using 4D dynamic segmentation algorithm, (4) Creation of averaged image, and (5) Creation of (REG) reconstruction using the averaged image.

proposed regularized (REG) reconstruction. The flowchart of calculations done to provide the (NR) and (REG) reconstructions is illustrated in Fig. 4.

To evaluate the performance of the new proposed algorithm, we defined three ROIs in the reconstruction: left kidney (LK), right kidney (RK) and bladder (BD), whose boundaries were known exactly from the phantom. For cases E and F, an extra ROI was included in the partially unhealthy kidney. For each ROI we measured the relative error of the summed TAC by

$$
\Delta A \%=\frac{\left\|T A C_{\text {recon }}-T A C_{\text {true }}\right\|_{2}}{\left\|T A C_{\text {true }}\right\|_{2}} \times 100 \%
$$

where $\|\cdot\|_{2}$ is the $l_{2}$-norm, $T A C_{\text {recon }}$ was obtained by summing the TACs in every voxel of that ROI in the reconstructed image, and $T A C_{\text {true }}$ was obtained similarly from the phantom. We calculated $\Delta A \%$ for both the (NR) and (REG) reconstructions. Two additional cases with incorrect segmentations were also tested - case $\mathrm{C}$ with two segments defined in the uniformly unhealthy left kidney, and case E with only one segment defined in the left kidney (which had healthy and unhealthy parts). In these cases, we wished to examine whether the algorithm produced poor results if the user made an incorrect assumption during segmentation; i.e., defining more or fewer segments than required. These two cases are referred to by (REG-W).

\section{RESULTS}

Results are summarized in Table II. For the kidney ROIs, the segmentation-based regularization reduced the error $\Delta A \%$ in every case. The improvement was especially noticeable in regions with healthy behaviour; the (REG) image had $\Delta A \%$ values more than five times smaller than those found in the (NR) image in 3 cases. The error for unhealthy TACs was reduced by 1.5 to 2.5 times, in general. The summed TAC for the bladder was not generally improved by the regularization; one possible explanation is that the bladder region in the phantom is about half the size of either of the kidneys, making it more difficult to obtain an accurate segmentation. Finally, in the two cases with incorrect segmentations (REG-W), the error was not appreciably reduced in the poorly segmented ROIs, illustrating the importance of using a good segmentation. It 

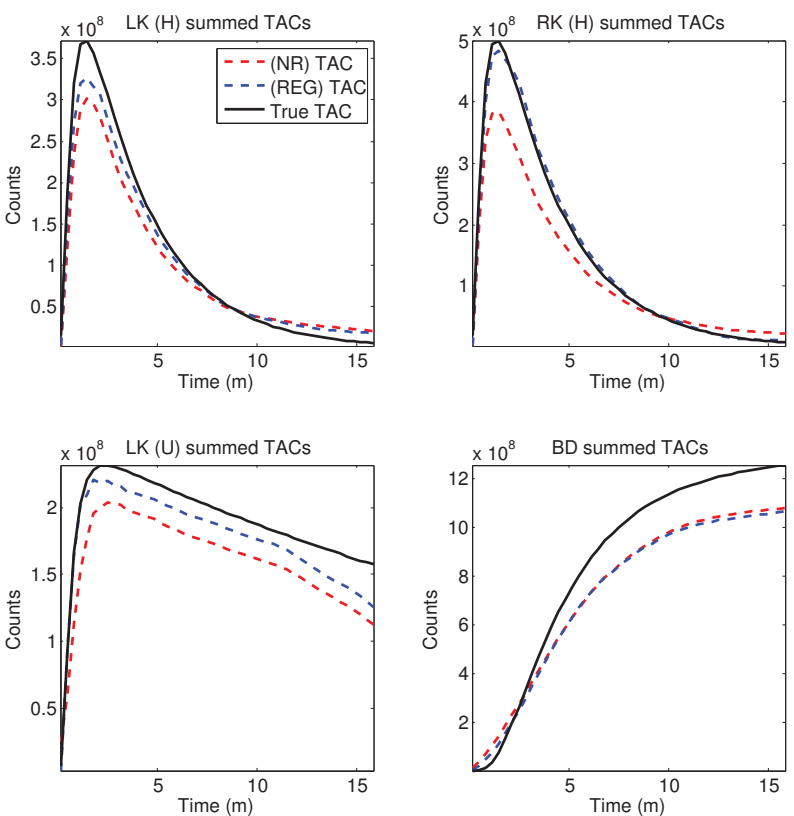

Fig. 5. Summed TACS generated for case E, showing typical improvements. The (REG) TAC is noticeably better for all ROIs except the bladder, where performance is the same as the (NR) case.

is worth noting, however, that accuracy was not diminished, even in case (E - REG-W) where the non-uniform left kidney was made uniform in the regularization step, prior to the second dSPECT run. Thus, the second dSPECT run was able to correct for this error.

Visually, the (REG) reconstructions are also an improvement on the (NR) reconstructions. The boundaries between the kidneys and background activity are generally more sharply delineated, with less blurring evident. Figure 6 shows an example of a coronal slice illustrating this improved image contrast.

\begin{tabular}{|l|c|c|c|c|c||}
\hline Simulation & RK(H) & RK (U) & LK (H) & LK (U) & BD \\
\hline A - NR & 24.0 & $\times$ & 19.8 & $\times$ & $\mathbf{1 4 . 0}$ \\
A - REG & $\mathbf{4 . 5}$ & $\times$ & $\mathbf{5 . 5}$ & $\times$ & 15.2 \\
\hline B - NR & $\times$ & 19.3 & $\times$ & 14.8 & 18.8 \\
B - REG & $\times$ & $\mathbf{5 . 6}$ & $\times$ & $\mathbf{5 . 3}$ & $\mathbf{7 . 1}$ \\
\hline C - NR & 25.4 & $\times$ & $\times$ & 14.6 & 14.9 \\
C - REG & 6.9 & $\times$ & $\times$ & $\mathbf{8 . 3}$ & $\mathbf{1 3 . 1}$ \\
C - REG-W & $\mathbf{5 . 3}$ & $\times$ & $\times$ & 13.6 & 15.4 \\
\hline D - NR & $\times$ & 18.8 & 19.7 & $\times$ & $\mathbf{1 4 . 7}$ \\
D - REG & $\times$ & $\mathbf{7 . 8}$ & $\mathbf{3 . 7}$ & $\times$ & 15.6 \\
\hline E - NR & 23.2 & $\times$ & 20.4 & 16.3 & $\mathbf{1 4 . 4}$ \\
E - REG & 4.5 & $\times$ & 11.5 & $\mathbf{7 . 8}$ & 15.2 \\
E - REG-W & $\mathbf{4 . 3}$ & $\times$ & $\mathbf{8 . 5}$ & 15.2 & 15.3 \\
\hline F - NR & 29.5 & 23.3 & 19.0 & $\times$ & 14.7 \\
F - REG & $\mathbf{1 2 . 5}$ & $\mathbf{1 4 . 7}$ & $\mathbf{1 3 . 4}$ & $\times$ & $\mathbf{1 3 . 9}$ \\
\hline
\end{tabular}

TABLE II

RELATIVE ERROR $\triangle A \%$ FOR THE THREE ROIS FOR EACH SIMULATION. THE BEST RESULT FOR EACH CASE IS HIGHLIGHTED IN BOLD. (H) AND

(U) DENOTE REGIONS OF HEALTHY AND UNHEALTHY BEHAVIOUR, WHERE APPLICABLE. FOR INSTANCE, IN (D) THE ENTIRE RIGHT KIDNEY

WAS UNHEALTHY AND THE ENTIRE LEFT KIDNEY WAS HEALTHY, SO RK(H) AND LK(U) DO NOT APPLY (DENOTED BY $\times$ ).
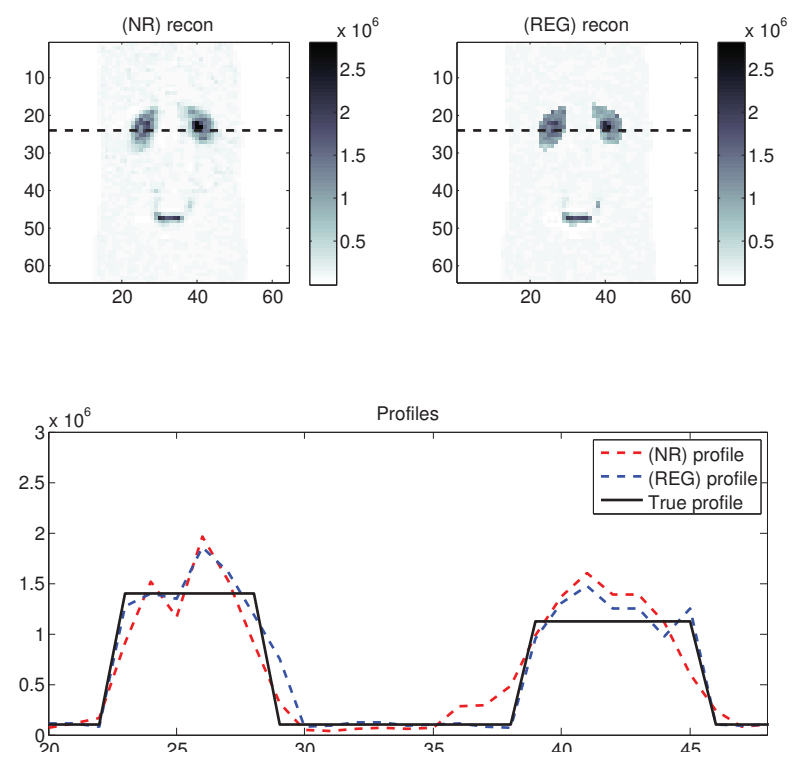

Fig. 6. Profiles drawn through kidneys in a single coronal slice for Case A, at a frame in the third minute. The (REG) reconstruction is less blurred and the profile agrees much better with the true profile at the organ edges than the $(\mathrm{NR})$ reconstruction.

\section{Conclusions}

In this paper we incorporate a user-assisted segmentation method into the dSPECT reconstruction algorithm. The segmentation is applied between two runs of the reconstruction algorithm in order to provide more spatial information to the reconstruction. We find that using segmentation as a means of spatial regularization can significantly improve the accuracy of the summed TACs within ROIs in the reconstruction, as well as image contrast. Furthermore, the algorithm is robust, in the sense that errors made during the segmentation step do not worsen the quality of the reconstruction.

\section{ACKNOWLEDGMENTS}

This work was supported by the Natural Sciences and Engineering Research Council (NSERC) of Canada.

\section{REFERENCES}

[1] T. Farncombe, A. Celler, D. Noll, J. Maeght, and R. Harrop, "Dynamic SPECT imaging using a single camera rotation (dSPECT)," IEEE Trans. Nucl. Sci., vol. 46, no. 4, pp. 1055-1061, Aug 1999.

[2] T. Farncombe, A. Celler, C. Bever, D. Noll, J. Maeght, and R. Harrop, "The incorporation of organ uptake into dynamic SPECT (dSPECT) image reconstruction," IEEE Trans. Nucl. Sci., vol. 48, no. 1, pp. 3-9, Feb 2001

[3] A. Saad, G. Hamarneh, T. Möller, and B. Smith, "Kinetic modeling based probabilistic segmentation for molecular images," LNCS - MICCAI, pp. 244-252, 2008.

[4] W. Segars, D. Lalush, and B. Tsui, "A realistic spline-based dynamic heart phantom," IEEE Trans. Nucl, Sci., vol. 46, no. 3, pp. 503-506, Jun 1999.

[5] L. Shepp and Y. Vardi, "Maximum likelihood reconstruction for emission tomography," IEEE Trans. Med. Imag., vol. MI-2, pp. 113-122, 1982.

[6] L. Grady, "Random walks for image segmentation," IEEE Trans. Pattern Analysis and Machine Intelligence, pp. 244-252, 2008.

[7] J. Kniss, R. Van Uitert, A. Stephens, G.-S. Li, T. Tasdizen, and C. Hansen, "Statistically quantitative volume visualization," in Visualization, 2005. VIS 05. IEEE, Oct. 2005, pp. 287-294. 